\title{
A MAXIMUM LIKELIHOOD APPROACH TO CORRELATION DIMENSION AND ENTROPY ESTIMATION
}

\author{
- E. Olofsen, J. Degoede and R. Heijungs \\ Department of Physiology, \\ University of Leiden, \\ P.O. Box 9604 , \\ 2300 RC Leiden, The Netherlands
}

(Email; DeGoede@rullf2.LeidenUniv.NL)

To obtain the correlation dimension and entropy from an experimental time series we derive estimators for these quantities together with expressions for their variances using a maximum likelihood approach. The validity of these expressions is supported by Monte Carlo simulations. We illustrate the use of the estimators with a local recording of atrial fibrillation obtained from a conscious dog.

1. Introduction. At present there is considerable interest in analysing experimental time series using methods from nonlinear dynamical systems theory. For a quantitative characterization of dynamical systems from a measured signal, algorithms have been developed to estimate the dimension spectrum and the generalized Kolmogorov entropies (Broggi, 1988; Schuster, 1988). These quantities can be used to differentiate between (quasi-) periodic, chaotic and stochastic processes. Time series from living organisms usually show irregular behaviour. It is interesting to determine whether the underlying dynamics is low-dimensional chaotic, because then it can in principle be modelled by a small set of deterministic nonlinear differential equations, implying that the irregularities are an intrinsic part of the process. Examples could be the brain activity and the functioning of the heart in both health and disease. The correlation integral method (Grassberger and Procaccia, 1983b; Takens, 1983) is widely used to estimate the correlation dimension and entropy. A sufficient condition for chaos is that the correlation entropy is positive. Thus to identify chaos, we need a measure of the uncertainty, with which the correlation entropy can be estimated. Although attention has been given to the statistical error of estimators of the correlation dimension (Denker and Keller, 1986; Ramsey and Yuan, 1989; Abraham et al., 1990; Theiler, $1990 \mathrm{a} ; 1990 \mathrm{~b}$ ), this is not so for estimators of the correlation entropy.

This paper is organized as follows. In Section 2, we describe the correlation integral method. In Section 3 we will derive estimators of the correlation 
dimension and entropy together with expressions of their uncertainties using the maximum likelihood approach. We demonstrate their validity by Monte Carlo simulations in Section 4. An example of the usage of the expressions is given in Section 5, for a recording of atrial fibrillation and, finally, some conclusions are given in Section 6.

2. The Correlation Integral. We start with a brief description of the correlation integral method, since it is the basis of our maximum likelihood approach. To characterize a dynamical system from a time series $x(t)$, first the phase space of the system is reconstructed with the method of time delayed coordinates (Takens, 1981; Packard et al., 1980), i.e. with $M$ vectors:

$$
\vec{x}\left(t_{i}\right)=\left[x\left(t_{i}\right), x\left(t_{i}+l \Delta t\right), \ldots, x\left(t_{i}+(d-1) l \Delta t\right)\right]
$$

where $\Delta t$ is the sample time, $d$ is the embedding dimension and $l$ is an appropriate lag. The correlation integral $C(r)$ is defined as:

$$
\left.C(r)=M^{-2} \text { (number of pairs }(i, j) \text { with }\left\|\vec{x}\left(t_{i}\right)-\vec{x}\left(t_{j}\right)\right\| \leqslant r\right)
$$

where $\|.$.$\| denotes a norm. It is assumed that distances, r$, between two randomly chosen points in phase space obey the cumulative probability distribution function (Grassberger and Procaccia, 1983b):

$$
P(r)=\phi r^{\nu}=\tilde{\phi} \exp \left(-d l \Delta t K_{2}\right) r^{v}
$$

and that $C(r) \sim P(r)$ for sufficiently small $r$ and large $M$. We also assume that $\phi$ and $\widetilde{\phi}$ do not depend on $r$ (see, however, Theiler, 1988) and that $\widetilde{\phi}$ does not depend on the embedding dimension. The correlation dimension $v$ is now given by (Eckmann and Ruelle, 1985):

$$
v=\lim _{r \rightarrow 0} \lim _{M \rightarrow \infty} \frac{\ln C(r)}{\ln r}
$$

if $d$ is large enough (Takens, 1981). The correlation entropy $K_{2}$ is given by:

$$
\lim _{r \rightarrow 0} \lim _{d \rightarrow \infty} \frac{1}{d} \lim _{M \rightarrow \infty} \ln C(r)=-l \Delta t K_{2} .
$$

With experimental data, a "scaling region" $\left[r_{l}, r_{u}\right]$ must be identified, for which the distribution (3) is valid. The dimension can be estimated by the slope of a straight line through a number of points $\left(\ln r_{i}, \ln C\left(r_{i}\right)\right)$ in the scaling region. The entropy can be estimated by observing the behaviour of $C(r)$ as a function of $d$ but using equation (5) directly may result in slow convergence. Therefore 
one usually studies the quotient of two correlation integrals, at embedding dimensions $d$ and $d+e$ (Grassberger and Procaccia, 1983a):

$$
\ln \left(\frac{C_{d}(r)}{C_{d+e}(r)}\right)=e l \Delta t K_{2}
$$

We will now show how to obtain maximum likelihood estimators for both $v$ and $K_{2}$, together with their asymptotic variances, under the assumption that the finite number of distances is the only source of error.

3. Maximum Likelihood Methods. For the correlation dimension, maximum likelihood estimators have been derived by Takens (1985) and Ellner (1988). In their approach, the distribution function (3) is normalized, such that $P\left(r_{n}\right)=1$, with the consequence that the correlation entropy cannot be determined.

Now suppose we have a sample that consists of $N$ independent distances, with $N_{l}$ in the interval $\left[0, r_{l}\right], N_{s}$ in $\left[r_{l}, r_{u}\right]$ and $N_{u}$ in $\left[r_{u}, 1\right]$. The likelihood function for this doubly censored set of data is (Kendall and Stuart, 1979):

$$
L(v, \rho)=A\left[\rho\left(\frac{r_{l}}{r_{u}}\right)^{v}\right]^{N_{l}} \prod_{i=1}^{N_{s}}\left[\rho v r_{u}^{-1}\left(\frac{r_{i}}{r_{u}}\right)^{(v-1)}\right][1-\rho]^{N_{u}}
$$

where $A$ is a permutation coefficient and $\rho=\phi r_{u}^{v}$ is used for convenience. We now consider the case where the sample consists of $N_{d}$ distances calculated at embedding dimension $d$, and $N_{d+e}$ distances calculated at embedding dimension $d+e$. We also assume that the $N_{d}$ and $N_{d+e}$ distances are independent. The likelihood function for this case, $L\left(v, \rho_{d}, \rho_{d+e}\right)$, is the production of the likelihood functions $L_{d}\left(v, \rho_{d}\right)$ and $L_{d+e}\left(v, \rho_{d+e}\right)$.

By solving the likelihood equations (Kendall and Stuart, 1979), we find the maximum likelihood estimators of the parameters $v, \rho_{d}$ and $\rho_{d+e}$. These are:

$$
\hat{v}=-\frac{N_{s, d}+N_{s, d+e}}{\sum_{i=1}^{N_{s, d}} \ln \left(\frac{r_{i}}{r_{u, d}}\right)+N_{l, d} \ln \left(\frac{r_{l, d}}{r_{u, d}}\right)+\sum_{j=1}^{N_{s, d+e}} \ln \left(\frac{r_{j}}{r_{u, d+e}}\right)+N_{l, d+e} \ln \left(\frac{r_{l, d+e}}{r_{u, d+e}}\right)}
$$

and:

$$
\hat{\rho}_{d}=\frac{N_{l, d}+N_{s, d}}{N_{d}}
$$

with a similar expression for $\hat{\rho}_{d+e}$. The maximum likelihood estimator of the correlation entropy is: 


$$
\hat{K}_{2}=\frac{\ln \left(\frac{\hat{\rho}_{d} r_{u, d+e}^{v \Delta}}{\hat{\rho}_{d+e^{r_{u, d} \Delta}}}\right)}{e l \Delta t}
$$

where we used equation (3) and the property that a function of maximum likelihood estimators is itself a maximum likelihood estimator.

The asymptotic variances of the dimension and entropy estimators are obtained by inverting the information matrix (Kendall and Stuart, 1979). We find:

$$
\operatorname{var}(\hat{v})=\frac{v^{2}}{N_{d} \rho_{d}\left(1-\left(\frac{r_{l, d}}{r_{u, d}}\right)^{v}\right)+N_{d+e} \rho_{d+e}\left(1-\left(\frac{r_{l, d+e}}{r_{u, d+e}}\right)^{v}\right)}
$$

and:

$$
\operatorname{var}\left(\hat{K}_{2}\right)=\frac{\frac{1-\rho_{d}}{N_{d} \rho_{d}}+\frac{1-\rho_{d+e}}{N_{d+e} \rho_{d+e}}+\operatorname{var}(\hat{v}) \ln ^{2}\left(\frac{r_{u, d}}{r_{u, d+e}}\right)}{(e l \Delta t)^{2}} .
$$

If $r_{u, d}=r_{u, d+e}$, then $\hat{v}$ and $\hat{K}_{2}$ are uncorrelated. Moreover, the estimator of the entropy is equivalent to equation (6) if one substitutes $r=r_{u, d}=r_{u, d+e}$ and if the correlation integrals are based on independent distances.

The maximum likelihood estimator of the correlation dimension calculated at a single embedding dimension reads:

$$
\hat{v}_{d}=\frac{-N_{s, d}}{\sum_{i=1}^{N_{s, d}} \ln \left(r_{i}\right)+N_{l, d} \ln \left(r_{l, d}\right)}
$$

Its asymptotic variance is given by:

$$
\operatorname{var}\left(\hat{v}_{d}\right)=\frac{v_{d}^{2}}{N_{d} \rho_{d}\left(1-\left(\frac{r_{l, d}}{r_{u, d}}\right)^{v}\right)} .
$$

These equations are slight generalizations of Ellner's results. Note that the expressions for the "double" correlation dimension [equation (8)] and entropy [equation (10)] are only meaningful if the "single" correlation dimensions $\hat{v}_{d}$ and $\hat{v}_{d+e}$ [equation (13)] do not significantly differ.

4. Monte Carlo Simulations. Monte Carlo methods can be used to investigate properties of the distributions of random variables. Here we will study the properties of the derived maximum likelihood estimators in two interesting 
cases: (1) using distances drawn directly from the proposed distribution [equation (3)]; and (2) using distances between points in reconstructed phase space, using time series of a (simulated) chaotic system.

4.1. Simulations using "ideal data". From 1000 Monte Carlo trials of the dimension and entropy estimates using simulated data, i.e. independent distances drawn from the distribution [equation (3)], the means and variances were estimated. These were compared with the specified $v$ and $K_{2}$ and the asymptotic variances. The estimators and the expressions for the asymptotic variances appear to be accurate if both $N_{l, d}+N_{s, d}$ and $N_{l, d+e}+N_{s, d+e}$ are larger than about 50 .

4.2. Simulations using time series. Time series were generated using the following classical examples:

1. The Hénon map, governed by the equations (Grassberger et al., 1988; Hénon, 1976):

$$
\begin{aligned}
& x_{n+1}=1-a x_{n}^{2}+b y_{n} \\
& y_{n+1}=x_{n}
\end{aligned}
$$

with $a=1.4$ and $b=0.3 . x_{0}$ and $y_{0}$ were chosen uniformly in $[-1,1]$ and $[-0.1,0.1]$, respectively. Initial conditions within this area cause the iterates to approach the attractor (Hénon, 1976). Literature values are: $v=1.22$ and $K_{2}=0.325$ (Grassberger and Procaccia, 1983a).

2. The logistic map, governed by the equation (Grassberger et al., 1988):

$$
x_{n+1}=1-a x_{n}^{2}
$$

For $a=2$, analytical results (Grassberger et al., 1988) are $v=1$ and $K_{2}=\ln 2 . x_{0}$ was chosen uniformly in $[-1,1]$, so that any $x_{0}$ is near the attractor.

3. The sine wave:

$$
x_{n}=\sin \left(\omega n+\omega_{0}\right)
$$

with $\omega=2 / \pi$, resulting in approximately 10 points per cycle and $\omega_{0}$ chosen uniformly in $[0,2 \pi]$. The values for $v$ and $K_{2}$ are 1 and 0 , respectively.

The $x$-variable was used to generate a time series of length $L(\Delta t=1)$; the first $L_{s}$ iterates were discarded to avoid transient effects.

4.2.1. Effects of correlations between distances. For the derivation of the maximum likelihood estimators of the correlation dimension and entropy and their variances, we had to assume that the distances used are independent. The question now arises how many distances are independent if we have $P$ 
independent points in phase space. Ellner (1988) states that these distances must be calculated from non-overlapping collections of point, so that:

$$
N \leqslant P / 2
$$

where $P \leqslant L-(d-1) l$. However, Theiler (1990) states that $N \leqslant P$ can be used. We performed Monte Carlo simulations to investigate the effects of correlated distances, using time series obtained from the Hénon map. Pairs of random indices for the vectors [equation (1)] were drawn and the distances between these vectors calculated with the supremum norm. For different values of $N$, the dimension and entropy were estimated 1000 times. The embedding dimensions were chosen rather small $(d=3$ and $e=1)$ because for higher values the number of distances and the length of the time series should also be increased, making this experiment very expensive with regard to computer time.

The averaged estimated dimension and entropies were plotted $v s \log N$ in Fig. 1. We see that the entropy estimates have large systematic errors, due to the low value of the embedding dimension. This however, is of no consequence
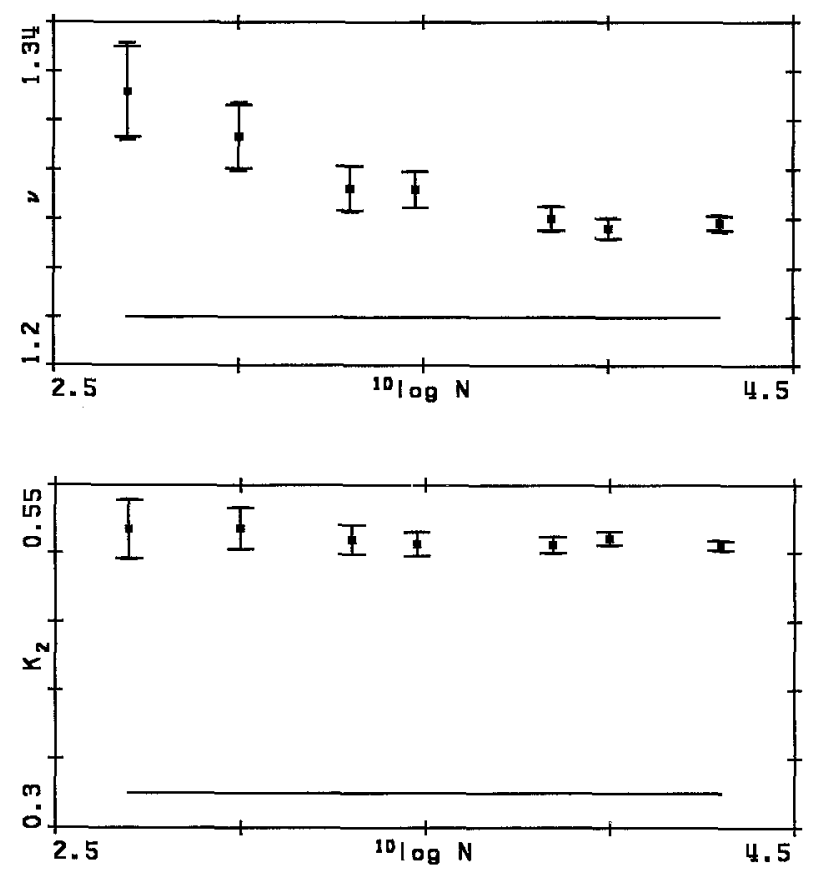

Figure 1. Averaged "single" dimension (upper panel) and entropy (lower panel) estimates with $95 \%$ confidence intervals (see text) as a function of $N$ for the Hénon map $\left(L=1000, d=3, e=1, l=1, r_{l}=0.01, r_{u}=0.07\right)$. The horizontal lines denote the literature values for the dimension and entropy. 
for our purpose to study the behaviour of the variances. We estimated $95 \%$ confidence intervals in two ways: (1) from the sample variance of the 1000 estimates (narrow bars); and (2) from the expressions of the asymptotic variances, but with averaged estimates of $v$ and $N_{l}+N_{s}$ (wide bars) since we do not know $\tilde{\phi}$. Increasing $N$ does decrease the fluctuations of the dimension and entropy estimates, but not as much as predicted by the formulas because the distances are becoming more and more correlated. From the figure we see that the variances are correct for values of $N$ between 1000 and 10000 . The lower bound arises from the fact that $N_{l}+N_{s}$ is getting below 50 . Correlations due to the deterministic nature of the dynamics are assumed to be of no consequence since for long time series the invariant probability measure on the attractor is approached. Furthermore, dynamical correlations in short time series can be suppressed by a method due to Theiler (1986).

4.2.2 Coverage frequencies. In this section we present results from Monte Carlo simulations, using time series from the given models, for different values of the embedding dimension. Furthermore, following Ellner, we computed coverage frequencies, i.e. the fractions of trials for which the confidence intervals:

$$
\begin{aligned}
\text { two-sided: } & {\left[\hat{q}-Z_{\alpha / 2} \sigma, \hat{q}+Z_{\alpha / 2} \sigma\right] } \\
\text { lower: } & {\left[\hat{q}-Z_{\alpha} \sigma, \infty\right] } \\
\text { upper: } & {\left[-\infty, \hat{q}+Z_{\alpha} \sigma\right] }
\end{aligned}
$$

contain the true values. Here $\hat{q}$ denotes a dimension or entropy estimate, $\sigma$ the square root of its (estimated) asymptotic variance, $Z_{\alpha}$ the probability- $\alpha$ critical value of the standard normal distribution and $\alpha$ the size of the test. For the size of a test we always used $\alpha=0.05$. The asymptotic variances were estimated by substitution of the estimated values of $v, \rho_{d}$ and $\rho_{d+e}$ in equations (14), (11) and (12). This procedure was repeated 100 times. The scaling regions were chosen by visual inspection, and justified by $X^{2}$ goodness-of-fit tests. Due to the supremum norm, the scaling regions hardly move to higher distance ranges as the embedding dimension increases, so we used the same $r_{l}$ and $r_{u}$ for every embedding dimension. Furthermore, we checked that there were no significant differences in successive "single" dimension estimates [equation (13)].

In Table 1 the results for the Hénon map are summarized. Noting that we have to divide the standard deviation by the square root of the number of Monte Carlo trials to obtain the standard error of the mean, we observe that the dimension estimates (Table $1 \mathrm{a}$ and $\mathrm{b}$ ) generally show small but statistically significant deviations from the literature value. Since $N_{l}+N_{s}$ is large enough, these systematic errors are not due to the use of the maximum likelihood estimator per sé, but to another source, probably lacunarity [non-constant $\tilde{\phi}$ 
Table 1(a). Averaged "single" dimension $(\overline{\hat{v}})$, standard deviation $(\widehat{\mathrm{SD}})$ and coverage frequency (CF) estimates for the Hénon map as functions of the embedding dimension. $\overline{N_{l}+N_{s}}$, averaged number of distances below $r_{u}\left(L=10000, L_{s}=1000, l=1, r_{l}=0.01\right.$, $r_{u}=0.07$ and $\left.N=100000\right)$

\begin{tabular}{rcccccc}
\hline$d$ & $\overline{\hat{v}}$ & $\widehat{\mathrm{SD}}(\hat{v})$ & Two-sided CF & Lower CF & Upper CF & $\overline{N_{t}+N_{s}}$ \\
\hline 1 & 0.942 & 0.009 & 0.00 & 1.00 & 0.00 & 15241.2 \\
2 & 1.195 & 0.018 & 0.66 & 0.99 & 0.48 & 6587.9 \\
3 & 1.256 & 0.021 & 0.64 & 0.45 & 1.00 & 4227.4 \\
4 & 1.203 & 0.026 & 0.89 & 1.00 & 0.80 & 2547.1 \\
5 & 1.186 & 0.028 & 0.77 & 1.00 & 0.69 & 1711.0 \\
6 & 1.201 & 0.035 & 0.94 & 0.98 & 0.91 & 1145.6 \\
7 & 1.199 & 0.044 & 0.93 & 0.99 & 0.88 & 789.8 \\
8 & 1.205 & 0.050 & 0.97 & 0.98 & 0.94 & 553.6 \\
9 & 1.201 & 0.065 & 0.95 & 0.98 & 0.92 & 388.8 \\
10 & 1.231 & 0.078 & 0.95 & 0.96 & 0.96 & 281.8 \\
11 & 1.235 & 0.095 & 0.96 & 0.93 & 0.95 & 194.8 \\
12 & 1.229 & 0.128 & 0.91 & 0.95 & 0.90 & 138.9 \\
13 & 1.199 & 0.150 & 0.89 & 0.94 & 0.89 & 95.4 \\
14 & 1.171 & 0.141 & 0.90 & 0.99 & 0.88 & 70.9 \\
15 & 1.206 & 0.177 & 0.96 & 0.97 & 0.93 & 51.0 \\
16 & 1.185 & 0.240 & 0.90 & 0.97 & 0.87 & 36.4 \\
17 & 1.213 & 0.244 & 0.93 & 1.00 & 0.88 & 26.1 \\
18 & 1.292 & 0.361 & 0.95 & 0.98 & 0.92 & 18.8 \\
19 & 1.272 & 0.415 & 0.93 & 0.98 & 0.87 & 14.1 \\
20 & 1.229 & 0.454 & 0.91 & 1.00 & 0.88 & 10.5 \\
\hline
\end{tabular}

Table 1(b). Averaged "double" dimension, standard deviation and coverage frequency estimates for the Hénon map; $e=1$

\begin{tabular}{rccccc}
\hline \multicolumn{1}{c}{$d$} & $\overline{\hat{v}}$ & $\widehat{\mathrm{SD}}(\hat{v})$ & Two-sided CF & Lower CF & Upper CF \\
\hline 4 & 1.202 & 0.020 & 0.87 & 0.99 & 0.81 \\
6 & 1.202 & 0.028 & 0.92 & 1.00 & 0.88 \\
8 & 1.213 & 0.043 & 0.95 & 0.98 & 0.92 \\
10 & 1.227 & 0.070 & 0.90 & 0.96 & 0.90 \\
12 & 1.204 & 0.093 & 0.89 & 0.97 & 0.88 \\
14 & 1.164 & 0.109 & 0.90 & 1.00 & 0.84 \\
\hline
\end{tabular}

(Theiler, 1988)]. In general, the two-sided coverage frequencies are too low and the lower and upper coverage frequencies are asymmetric; these should all be $0.95 \pm 0.02$. Since the asymptotic variances are accurate, these results are most probably due to systematic errors in the estimated correlation dimension. This is further illustrated by the fact that if we use the mean dimension estimate from the Monte Carlo trials as the "true" value, the coverage frequencies fluctuate closely around 0.95 . We emphasize that in contrast to Ellner's numerical results 
(Ellner, 1988), our computed confidence intervals are not "conservative". The entropy estimates converge to the literature value rather slowly (Table 1c and d). In Table $2 a$ we present the results for the logistic map. Note that the number

Table 1(c). Averaged entropy $\left(\hat{K}_{2}\right)$, standard deviation and coverage frequency estimates for the Hénon map; $e=1$

\begin{tabular}{rccccc}
\hline$d$ & $\widehat{\hat{K}_{2}}$ & $\widehat{\mathrm{SD}}\left(\hat{K}_{2}\right)$ & Two-sided CF & Lower CF & Upper CF \\
\hline 4 & 0.3980 & 0.0311 & 0.33 & 0.23 & 1.00 \\
6 & 0.3718 & 0.0496 & 0.79 & 0.70 & 0.98 \\
8 & 0.3535 & 0.0728 & 0.90 & 0.87 & 0.95 \\
10 & 0.3709 & 0.1061 & 0.89 & 0.86 & 0.98 \\
12 & 0.3785 & 0.1551 & 0.90 & 0.87 & 0.95 \\
14 & 0.3370 & 0.2034 & 0.96 & 0.94 & 0.95 \\
\hline
\end{tabular}

Table 1(d). Averaged entropy, standard deviation and coverage frequency estimates for the Hénon map; $e=3$

\begin{tabular}{rccccc}
\hline$d$ & $\hat{K}_{2}$ & $\widehat{\mathrm{SD}}\left(\hat{K}_{2}\right)$ & Two-sided CF & Lower CF & Upper CF \\
\hline 4 & 0.3905 & 0.0131 & 0.00 & 0.00 & 1.00 \\
6 & 0.3604 & 0.0210 & 0.53 & 0.45 & 0.99 \\
8 & 0.3490 & 0.0325 & 0.87 & 0.78 & 0.97 \\
10 & 0.3627 & 0.0450 & 0.86 & 0.79 & 1.00 \\
\hline
\end{tabular}

of distances used did not satisfy equation (18). Nevertheless the results for the coverage frequencies indicate that the variance are precise. Similar simulations, in which equation (18) was satisfied, do not yield better coverage frequencies as can be seen from Table $2 \mathrm{~b}$. Table 3 shows the results for the sine wave: the estimated entropies do not significantly differ from zero, except at embedding dimension 4.

Table 2(a). Averaged entropy, standard deviation and coverage frequency estimates for the logistic map $\left(L=2000000, L_{s}=1000, l=1, r_{l}=0.001, r_{u}=0.005, N=1000000\right.$ and $\left.e=1\right)$

\begin{tabular}{cccccc}
\hline$d$ & $\bar{K}_{2}$ & $\widehat{\mathrm{SD}}\left(\hat{K}_{2}\right)$ & Two-sided CF & Lower CF & Upper CF \\
\hline 4 & 0.6774 & 0.0455 & 0.92 & 0.98 & 0.88 \\
6 & 0.6743 & 0.0915 & 0.94 & 0.97 & 0.92 \\
8 & 0.7172 & 0.1902 & 0.95 & 0.93 & 0.95 \\
\hline
\end{tabular}


Table 2(b). Averaged entropy, standard deviation and coverage frequency estimates for the logistic map $\left(L=100000, L_{s}=1000, l=1, r_{l}=0.001, r_{u}=0.005, N=1000000\right.$ and $\left.e=1\right)$

\begin{tabular}{cccccc}
\hline$d$ & $\hat{K}_{2}$ & $\widehat{\mathrm{SD}}\left(\hat{K}_{2}\right)$ & Two-sided CF & Lower CF & Upper CF \\
\hline 4 & 0.6787 & 0.0449 & 0.95 & 0.97 & 0.90 \\
6 & 0.6891 & 0.0780 & 0.97 & 0.98 & 0.97 \\
8 & 0.7063 & 0.1990 & 0.91 & 0.91 & 0.96 \\
\hline
\end{tabular}

Table 3. Averaged entropy, standard deviation and coverage frequency estimates for the sine wave $\left[L=10000, L_{s}=0, l=3\right.$; with Theiler correction 3 (Theiler, 1986). $r_{l}=0.01, r_{u}=0.07$, $N=10000$ and $e=1]$

\begin{tabular}{rrrccc}
\hline$d$ & \multicolumn{1}{c}{$\hat{K}_{2}$} & $\widehat{\mathrm{SD}}\left(\hat{K}_{2}\right)$ & Two-sided CF & Lower CF & Upper CF \\
\hline 4 & 0.0056 & 0.0211 & 0.96 & 0.92 & 0.97 \\
6 & 0.0002 & 0.0218 & 0.93 & 0.94 & 0.93 \\
8 & -0.0042 & 0.0212 & 0.96 & 0.95 & 0.94 \\
\hline
\end{tabular}

5. An Application. Atrial fibrillation is a commonly encountered arrhythmia of the heart. Understanding its nature is an important issue in clinical cardiology. To study the underlying dynamics, recordings of induced fibrillation were collected from chronically instrumented conscious dogs with 30 unipolar electrodes sutured to the atria (see Rensma et al., 1988, for experimental details). Time series consisting of approximately 4000 points were obtained using an 8-bit analog-to-digital converter at a sampling frequency of $1000 \mathrm{~Hz}$. The time lag for the reconstruction of phase space was determined by the mutual information criterion (Fraser and Swinney, 1986), which yielded $l=21$. See Fig. 2 for plots of the time series and a phase portrait. The correlation dimension and entropy were estimated from 40000 distances (from randomly chosen vector indices). We set the Theiler correction parameter (Theiler, 1986) $W$ to 21 . We used $e=3$ in equation (10) to reduce its variance. The scaling region was $[0.061,0.198]$ so $r_{u} / r_{l}$ is about 3 . The results are presented in Fig. 3. We see that both the dimension and entropy estimates converge. The "double" correlation dimension and the correlation entropy for $d=11$ and $e=9$ are $\hat{v}=2.6 \pm 0.1$ and $\hat{K}_{2}=9.7 \pm 0.8$ [nats s$^{-1}$ ]. These results suggest that this particular episode of fibrillation may be characterized by a low-dimensional chaotic process. To check whether the saturation is not caused by autocorrelated or coloured noise, we randomized the phases of the signal (Theiler, 1990a). For this "randomized" signal, the estimated correlation dimension does not saturate with increasing embedding dimension. Increasing the Theiler correction parameter $W$ hardly changes our results. To create a 

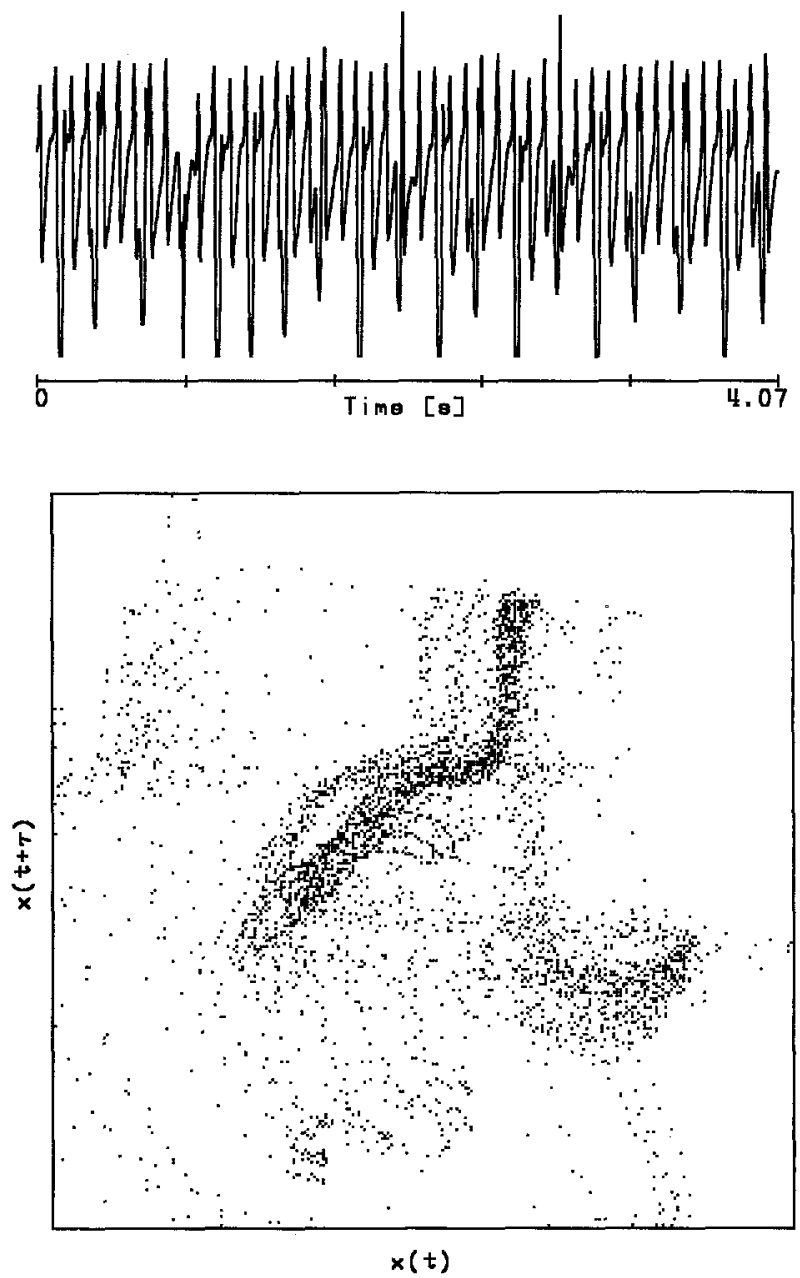

Figure 2. Plot of the recording of atrial fibrillation (upper panel) and a phase portrait (lower panel); $\tau=l \Delta t, l=21, \Delta t=0.001$.

time series that mimicks the fibrillation data we used the $x$-component of the Rössler system (see Wolf et al., 1985). For parameter values $L=4000$, $\Delta t=0.06, l=W=22,\left[r_{l}, r_{u}\right]=[0.029,0.102], d=11$ and $e=9$, we found for the "double" correlation dimension and entropy $\hat{v}=2.2 \pm 0.1$ and $\hat{K}_{2}=0.10 \pm 0.01$ [nats s${ }^{-1}$ ] which is in reasonable agreement with the literature values. We remark that the Rössler time series was only corrupted by a small amount of "integration noise". Work is in progress with longer experimental electrograms and time series from model systems to study both the effects of noise and methods of noise reduction (Kostelich and Yorke, 1990) on the estimated correlation dimension and entropy. 

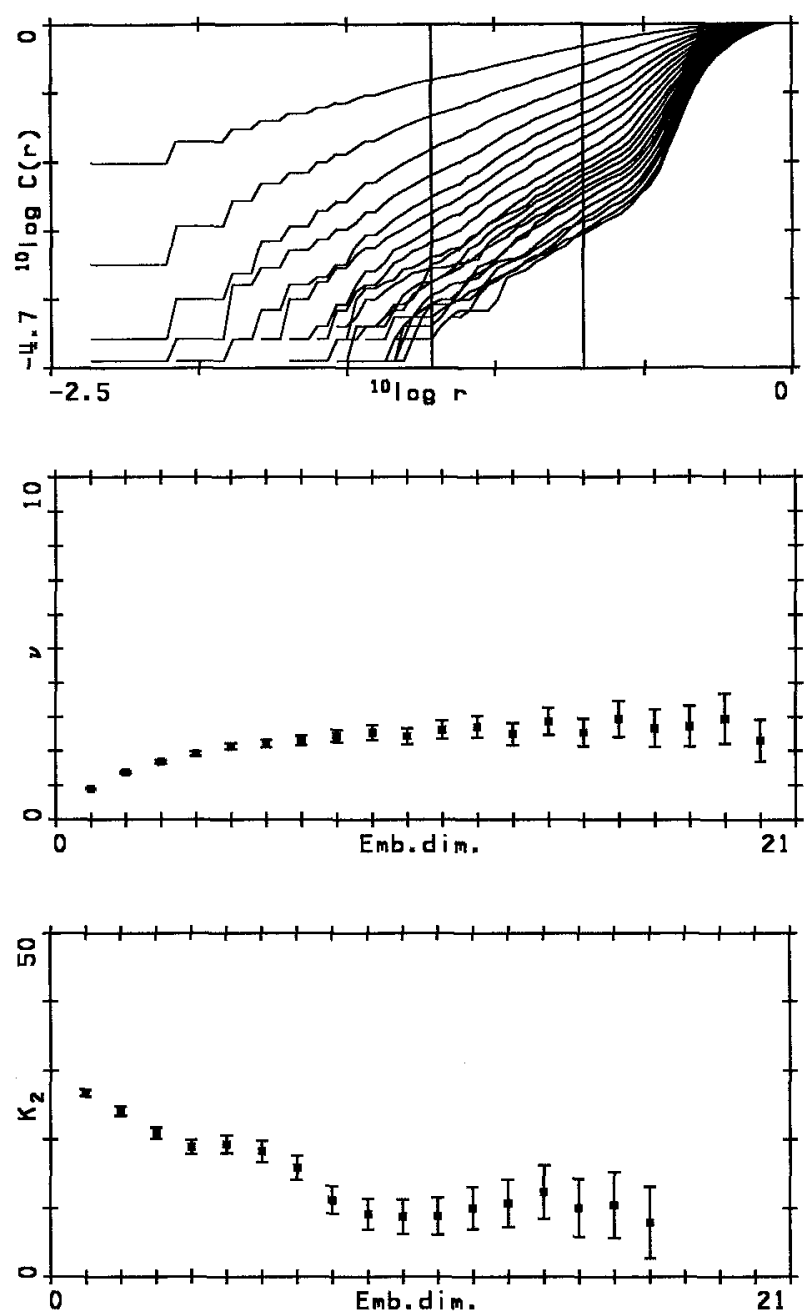

Figure 3. Correlation integral $C(r)$ vs $r$ for the recording of atrial fibrillation (upper panel). The values for $d$ are 1 (top curve), 2, ., 20 (bottom curve). The vertical lines are the boundaries of the scaling regions. Estimated correlation dimensions (middle panel) and entropies (lower panel) with $95 \%$ confidence intervals.

6. Conclusions. Our numerical results from the simulated data indicate that the expressions for the asymptotic variances are reliable. In combination with mathematical models for which the correlation dimension and entropy are known theoretically these expressions can be used to identify systematic errors and limitations of the dimension and entropy estimators in general; in applications they can be useful to identify chaos. To obtain the most precise estimates for the correlation dimension and the correlation entropy, the maximum likelihood method suggests to choose the largest possible values for 
$r_{u}$ and for $e$. We remark that our expressions are also valid if the scaling regions are not the same at different embedding dimensions, which occurs if one uses the Euclidean norm. A potential disadvantage of this maximum likelihood approach is that the distances used must be independent. This is almost unavoidable in order to have a simple enough likelihood function.

The results obtained by applying the derived estimators to the electrogram recorded from the atrium of a conscious dog suggest that some types of atrial fibrillation may be characterized by low-dimensional chaotic dynamics. As aptly pointed out by Ruelle (1990) there is a real danger that the present methods for detecting chaos are applied beyond their domain of validity. However, in our application to the atrial fibrillation data the time series and the scaling region seem long enough for the estimation of a low value of the correlation dimension. Of course to draw firm conclusions about the dynamics of experimental time series in general and atrial fibrillation in particular much more work has to be done.

We are greatly indebted to Professors R. D. Gill and W. R. van Zwet for suggestions and reading the manuscript and to Professor M. A. Allessie and his group for providing the electrograms.

\section{LITERATURE}

Abraham, N. B., A. M. Albano, A. Passamante and P. E. Rapp (Eds). 1990. Measures of Complexity and Chaos. New York: Plenum Press.

Broggi, G. 1988. Evaluation of dimensions and entropies of chaotic systems. J. opt. Soc. Am. B5, 1020-1028.

Denker, M. and G. Keller. 1986. Rigorous statistical procedures for data from dynamical systems. J. stat. Phys. 44, 67-93.

Eckmann, J.-P. and D. Ruelle. 1985. Ergodic theory of chaos and strange attractors. Rev. mod. Phys. 57, 617-656.

Ellner, S. 1988. Estimating attractor dimensions from limited data: a new method, with error estimates. Phys. Lett. A133, 128-133.

Fraser, A. M. and H. L. Swinney. 1986. Independent coordinates for strange attractors from mutual information. Phys. Rev. A33, 1134-1140.

Grassberger, P. and I. Procaccia. 1983a. Estimation of the Kolmogorov entropy from a chaotic signal. Phys. Rev. A28, 2591-2593.

Grassberger, P. and I. Procaccia. 1983b. Measuring the strangeness of strange attractors. Physica 9D, 189-207.

Grassberger, P., R. Badii and A. Politi. 1988. Scaling laws for invariant measures on hyperbolic and nonhyperbolic attractors. J. stat. Phys. 51, 135-178.

Hénon, M. 1976. A two-dimensional mapping with a strange attractor. Commun. math. Phys. 50, 69-77.

Kendall, M. G. and A. Stuart. 1979. The Advanced Theory of Statistics, Vol. 2. London: Griffin.

Kostelich, E. J. and J. A. Yorke. 1990. Noise reduction: finding the simplest dynamical system consistent with the data. Physica D41, 183-196.

Packard, N. H., J. P. Crutchfield, J. D. Farmer and R. S. Shaw. 1980. Geometry from a time series. Phys. Rev. Lett. 45, 712-716. 
Ramsey, J. B. and H.-J, Yuan. 1989. Bias and error bars in dimension calculations and their evaluation in some simple models. Phys. Lett. A134, 287-297.

Rensma, P. L., M. A. Allessie, W. J. E. P. Lammers, F. I. M. Bonke and M. J. Schalij. 1988. The length of the excitation wave and the susceptibility to reentrant atrial arrhythmias in normal conscious dogs. Circ. Res. 62, 395-410.

Ruelle, D. 1990. Deterministic chaos: the science and the fiction. Proc. R. Soc. Lond. A427, 241-248.

Schuster, H. G. 1988. Deterministic Chaos, an Introduction. Weinheim: VCH.

Takens, F. 1981. Detecting strange attractors in turbulence. In Lecture Notes in Mathematics, Vol. 898, pp. 366-381. Berlin: Springer.

Takens, F. 1983. Invariants related to dimension and entropy. In Atas do $13^{\circ}$. Rio de Janeiro: Colóqkio Brasiliero do Matemática.

Takens, F. 1985. On the numerical determination of the dimension of an attractor. In Lecture Notes in Mathematics, Vol. 1125, pp. 99-106. Berlin: Springer.

Theiler, J. 1986. Spurious dimension from correlation algorithms applied to limited time-series data. Phys. Rev. A34, 2427-2432.

Theiler, J. 1988. Lacunarity in a best estimator of fractal dimension. Phys. Lett. A133, 195-200.

Theiler, J. 1990a. Estimating fractal dimension. J. opt. Soc. Am. A7, 1055-1073.

Theiler, J. 1990b. Statistical precision of dimension estimators. Phys. Rev. A41, 3038-3051.

Wolf, A., J. B. Swift, H. L. Swinney and J. A. Vastano. 1985. Determining Lyapunov exponents from a time series. Physica 16D, 285-317. 